\title{
Réunions et Voyage d'Études du Groupe de Travail de Correction des Torrents et de Lutte contre les Avalanches
}

\author{
Organisés par le Gouvernement français, sous l'égide de l’O.N.U., dans les Alpes françaises, \\ du 28 juin au 8 juillet 1952
}

\section{Training of mountain torrents and battle against avalanches}

\author{
PAR P. RENEUNE \\ CONSERVATEUR DES FAUX ET FORÊTS \\ A I'ÉCOLE Nationale DES EavX ET FOREtTs, NANCY
}

ET

INGÉNIEUR AU LABORATOIRE NATIONAL D'HYDRAULIQUE GHATOU

English synopsis, p. 113

\section{VUES D'ENSEMBLE}

\section{Le groupe de travail}

La Commission Europécnne des Forêts et Produits Forestiers, organe de la Division des Forêts de la F.A.O., dans l'Organisation des Nations Unies, avait recommandé lors de sa $4^{\circ}$ session, en 1951, de constituer un Groupe de 'Travail de la Correction des Torrents et de la lutte contre les Avalanches; elle tient en effet pour essentiels les problèmes de la lutte contre l'́rosion en montagne, que les forestiers francais s'occupent de résoudre depuis près d'un siècle, le rôle de la végétation ligneuse y étant prédominant.

La premiere manifestation de ce Groupe a eu lieu, dans les Alpes Francaises, du 28 juin au 8 juillet 1952, favorisce par un ciel exceptionnellement tenace dans la générosité de sa lumiere et de ses calories. Organisée par le Gouvernement français, clle a comporté un voyage d'ctudes ouvert à Nice, coupé d'une séance de travail on salle à Briancon et cloturé à Evian. Ainsi se trouvait matérialisć le double aspect fondamental et classique du massif alpin français, le méditerranéen des Alpes Sèches du Sud et celui des Savoies dans les Alpes Vertes du Nord.

Le Groupe comprenait des représentants et observateurs de onze pays: Allemagne, Autriche, Espagne, France, Grande-Bretagne (Chypre), Iran, Italie, Maroc, Suisse, Turquie et Yougoslavie, qui avaient délégué leurs spécialistes forestiers et hydrauliciens, ceux des deux dernières nations étant anciens élèves de l'Ecole Forestière à Nancy.

Le Groupe de Travail a considéré que son mandat consistait essentiellement dans l'étude des problèmes posés, dans les régions montagneuses de l'Europe à forte densité de population, par la défense des villages, des terrains cultivés, des agglomérations industrielles et des ouvrages hydro-électriques, contre les torrents et les avalanches, tant pour la conservation des sols en montagne que pour le maintien des populations montagnardes avec des conditions de vie acceptables. 